\title{
A Simple but Powerful Automatic Term Extraction Method
}

\author{
Hiroshi Nakagawa \\ Information Technology Center, \\ The University of Tokyo \\ 7-3-1, Bunkyo, Hongo \\ Tokyo, Japan, 113-0033 \\ nakagawa@r.dl.itc.u-tokyo.ac.jp
}

\begin{abstract}
In this paper, we propose a new idea for the automatic recognition of domain specific terms. Our idea is based on the statistics between a compound noun and its component single-nouns. More precisely, we focus basically on how many nouns adjoin the noun in question to form compound nouns. We propose several scoring methods based on this idea and experimentally evaluate them on the NTCIR1 TMREC test collection. The results are very promising especially in the low recall area.
\end{abstract}

\section{Introduction}

Automatic term recognition, ATR in short, aims at extracting domain specific terms from a corpus of a certain academic or technical domain. The majority of domain specific terms are compound nouns, in other words, uninterrupted collocations. $85 \%$ of domain specific terms are said to be compound nouns. They include single-nouns of the remaining $15 \%$ very frequently as their components, where "single-noun" means a noun which could not be further divided into several shorter and more basic nouns. In other words, the majority of compound nouns consist of the much smaller number of the remaining $15 \%$ single-noun terms and other single-nouns. In this situation, it is natural to pay attention to the relation among single-nouns and compound nouns, especially how single-noun terms contribute to make up compound noun terms.

Another important feature of domain

\author{
Tatsunori Mori \\ Yokohama National University \\ 79-5, Tokiwadai,Hodogaya \\ Yokohama, Japan,240-0085 \\ mori@forest.dnj.ynu.ac.jp
}

specific terms is termhood proposed in (Kageura \& Umino 96) where "termhood" refers to the degree that a linguistic unit is related to a domain-specific concept. Thus, what we really have to pursue is an ATR method which directly uses the notion of termhood.

Considering these factors, the way of making up compound nouns must be heavily related to the termhood of the compound nouns. The first reason is that termhood is usually calculated based on term frequency and bias of term frequency like inverse document frequency. Even though these calculations give a good approximation of termhood, still they are not directly related to termhood because these calculations are based on superficial statistics. That means that they are not necessarily meanings in a writer's mind but meanings in actual use. Apparently, termhood is intended to reflect this type of meaning. The second reason is that if a certain single-noun, say $N$, expresses the key concept of a domain that the document treats, the writer of the document must be using $N$ not only frequently but also in various ways. For instance, he/she composes quite a few compound nouns using $N$ and uses these compound nouns in documents he/she writes. Thus, we focus on the relation among single-nouns and compound nouns in pursuing new ATR methods.

The first attempt to make use of this relation has been done by (Nakagawa $\&$ Mori 98) through the number of distinct single-nouns that come to the left or right of a single-noun term when used in compound noun terms. Using this type of number associated with a single-noun 
term, Nakagawa and Mori proposed a scoring function for term candidates. Their term extraction method however is just one example of employing the relation among single-nouns and compound nouns. Note that this relation is essentially based on a noun bigram. In this paper, we expand the relation based on noun bigrams that might be the components of longer compound nouns. Then we experimentally evaluate the power of several variations of scoring functions based on the noun bigram relation using the NTCIR1 TMREC test collection. By this experimental clarification, we could conclude that the single-noun term's power of generating compound noun terms is useful and essential in ATR.

In this paper, section 1 gives the background of ATR methods. Section 2 describes the proposed method of the noun bigram based scoring function for term extraction. Section 3 describes the experimental results and discusses them.

\section{Background}

\subsection{Candidates Extraction}

The first thing to do in ATR is to extract term candidates from the given text corpus. Here we only focus on nouns, more precisely a single-noun and a compound noun, which are exactly the targets of the NTCIR1 TMREC task(Kageura et al 1999). To extract compound nouns which are promising term candidates and at the same time to exclude undesirable strings such as "is a" or "of the", the frequently used method is to filter out the words that are members of a stop-word-list. More complex structures like noun phrases, collocations and so on, become focused on (Frantzi and Ananiadou 1996). All of these are good term candidates in a corpus of a specific domain because all of them have a strong unithood (Kageura\&Umino96) which refers to the degree of strength or stability of syntagmatic combinations or collocations. We assume the following about compound nouns or collocations:
Assumption Terms having complex structure are to be made of existing simple terms

The structure of complex terms is another important factor for automatic term candidates extraction. It is expressed syntactically or semantically. As a syntactic structure, dependency structures that are the results of parsing are focused on in many works. Since we focus on these complex structures, the first task in extracting term candidates is a morphological analysis including part of speech (POS) tagging. For J apanese, which is an agglutinative language, a morphological analysis was carried out which segmented words from a sentence and did POS tagging (Matsumoto et al. 1996).

After POS tagging, the complex structures mentioned above are extracted as term candidates. Previous studies have proposed many promising ways for this purpose, Hisamitsu(2000) and Nakagawa (1998) concentrated their efforts on compound nouns. Frantzi and Ananiadou (1996) tried to treat more general structures like collocations.

\subsection{Scoring}

The next thing to do is to assign a score to each term candidate in order to rank them in descending order of termhood. Many researchers have sought the definition of the term candidate's score which approximates termhood. In fact, many of those proposals make use of surface statistics like tf · idf. Ananiadou et al. proposed C-value (Frantzi and Ananiadou 1996) and NC-value (Frantzi and Ananiadou 1999) which count how independently the given compound noun is used in the given corpus. Hisamitsu (2000) propose a way to measure termhood that counts how far the given term is different from the distribution of non-domain-specific terms. All of them tried to capture how important and independent a writer regards and uses individual terms in a corpus 
2 Single-Noun Bigrams as Components of Compound Nouns

\subsection{Single-Noun Bigrams}

The relation between a single-noun and complex nouns that include this singlenoun is very important. Nevertheless, to our knowledge, this relation has not been paid enough attention so far. Nakagawa and Mori (1998) proposed a term scoring method that utilizes this type of relation. In this paper, we extend our idea comprehensively. Here we focus on compound nouns among the various types of complex terms. In technical documents, the majority of domain-specific terms are noun phrases or compound nouns consisting of a small number of single nouns. Considering this observation, we propose a new scoring method that measures the importance of each single-noun. In a nutshell, this scoring method for a single-noun measures how many distinct compound nouns contain a particular single-noun as their part in a given document or corpus. Here, think about the situtation where single-noun $\mathrm{N}$ occurs with other singlenouns which might be a part of many compound nouns shown in Figure 1 where [N M] means bigram of noun $\mathrm{N}$ and $\mathrm{M}$.

$$
\begin{gathered}
{\left[\begin{array}{cc}
L N 1 & N
\end{array}\right](\text { ( 1) }} \\
: \\
{\left[\begin{array}{ll}
L N n & N
\end{array}\right](L N)}
\end{gathered}
$$

$$
\begin{aligned}
& \text { [N RN1](\#R1) } \\
& \text { [N RNm] (\#Rm) }
\end{aligned}
$$

Figure 1. Noun Bigram and their Frequency

In Figure 1, [LNi N] $(\mathrm{i}=1, . ., \mathrm{n})$ and [N $R N j](j=1, \ldots, m)$ are single-noun bigrams which constitute (parts of) compound nouns. \# $i$ and \#Rj $(i=1, . ., n$ and $j=1, . ., m)$ mean the frequency of the bigram [LNi $\mathrm{N}$ ] and [N RNj] respectively. Note that since we depict only bigrams, compound nouns like [L Ni N RNj] which contains [ [ $\left.\begin{array}{ll}\mathrm{Ni} & \mathrm{N}\end{array}\right]$ and/or [N RNj] as their parts might actually occur in a corpus. Again this noun trigram might be a part of longer compound nouns.
Let us show an example of a noun bigram. Suppose that we extract compound nouns including "trigram" as candidate terms from a corpus shown in the following example.

Example 1.

trigram statistics, word trigram, class trigram, word trigram, trigram acquisition, word trigram statistics, character trigram

Then, noun bigrams consisting of a single-noun "trigram" are shown in the following where the number bewteen ( and ) shows the frequency.

word trigram (3) trigram statistics (2) class trigram (1) trigram acquisition (1) character trigram(1)

Figure 2. An example of noun bigram

We just focus on and utilize singlenoun bigrams to define the function on which scoring is based. Note that we are concerned only with single-noun bigrams and not with a single-noun per se. The reason is that we try to sharply focus on the fact that the majority of domain specific terms are compound nouns. Compound nouns are well analyzed as noun bigram.

\subsection{Scoring Function}

\subsubsection{The direct score of a noun bigram}

Since a scoring function based on [LNi N] or [N RNj] could have an infinite number of variations, we here consider the following simple but representative scoring functions.

\#LDN(N) and \#RDN(N): These are the number of distinct single-nouns which directly precede or succeed $\mathbf{N}$. These are exactly " $n$ " and " $m$ " in Figure 1. For instance, in an example shown in Figure 2, \#LDN (trigram)=3, \#RDN (trigram) $=2$

$\operatorname{LN}(\mathrm{N}, \mathrm{k})$ and $\mathrm{RN}(\mathrm{N}, \mathrm{k})$ : The general functions that take into account the number of occurrences of each noun bigram like [ $\mathrm{LNi} \mathrm{N}]$ and [N RNj] are defined as follows. 


$$
\begin{aligned}
& \mathbf{L N}(\mathbf{N}, \mathbf{k})=\sum_{\mathbf{i}=\mathbf{1}}^{\# \mathbf{L D N}(\mathbf{N})}(\# \mathbf{L i})^{\mathbf{k}} \\
& \mathbf{R N}(\mathbf{N}, \mathbf{k})=\sum_{\mathbf{j}=\mathbf{1}}^{\# \mathbf{R D N}(\mathbf{N})}(\# \mathbf{R j})^{\mathbf{k}}
\end{aligned}
$$

We can find various functions by varying parameter $k$ of (1) and (2). For instance, \#DN $(\mathrm{N})$ and \#RDN(N) can be defined as $\operatorname{LN}(N, 0)$ and $R N(N, 0)$. $\operatorname{LN}(N, 1)$ and $\mathrm{RN}(\mathrm{N}, 1)$ are the frequencies of nouns that directly precede or succeed $\mathrm{N}$. In the example shown in Figure 2, for example, $\mathrm{LN}($ trigram, 1$)=5$, and RN (trigram, 1$)=3$. Now we think about the nature of (1) and (2) with various value of the parameter $k$. The larger $k$ is, the more we take into account the frequencies of each noun bigram. One extreme is the case $k=0$, namely $\operatorname{LN}(\mathrm{N}, 0)$ and $\operatorname{RN}(\mathrm{N}, 0)$, where we do not take into account the frequency of each noun bigram at all. $\operatorname{LN}(N, 0)$ and $\mathrm{RN}(\mathrm{N}, 0)$ describe how linguistically and domain dependently productive the noun $\mathrm{N}$ is in a given corpus. That means that noun $\mathrm{N}$ presents a key and/or basic concept of the domain treated by the corpus. Other extreme cases are large k, like $k=2,4$, etc. In these cases, we rather focus on frequency of each noun bigram. In other words, statistically biased use of noun $\mathrm{N}$ is the main concern. In the example shown in Figure 2, for example, $\mathrm{LN}($ trigram, 2$)=11$, and RN (trigram,2) $=5$. If $k<0$, we discount the frequency of each noun bigram. However, this case does not show good results of in our ATR experiment.

\subsubsection{Score of compound nouns}

The next thing to do is to extend the scoring functions of a single-noun to the scoring functions of a compound noun. We adopt a very simple method, namely a geometric mean. Now think about a compound noun : $\mathrm{CN}=\mathrm{N}_{1} \mathrm{~N}_{2} \ldots \mathrm{N}$ L. Then a geometric mean: $\mathrm{GM}$ of $\mathrm{CN}$ is defined as follows.

$\mathrm{GM}(\mathrm{CN}, \mathrm{k})$

$$
=\left(\prod_{\mathrm{i}=1}^{\mathrm{L}}\left(\mathrm{LN}\left(\mathrm{N}_{\mathrm{i}}, \mathrm{k}\right)+1\right)\left(\mathrm{RN}\left(\mathrm{N}_{\mathrm{i}}, \mathrm{k}\right)+1\right)\right)^{1 / 2 \mathrm{~L}}-(3)
$$

For instance, if we use $\operatorname{LN}(N, 1)$ and $\mathrm{RN}(\mathrm{N}, 1)$ in example $1, \mathrm{GM}($ trigram, 1$)=$ $\sqrt{(3+1) \times(5+1)}=4.90$. In (3), GM does not depend on the length of a compound noun that is the number of single-nouns within the compound noun. This is because we have not yet had any idea about the relation between the importance of a compound noun and a length of the compound noun. It is fair to treat all compound nouns, including single-nouns, equally no matter how long or short each compound noun is.

\subsubsection{Combining Compound Noun Frequency}

Information we did not use in the bigram based methods described in 2.2.1 and 2.2.2 is the frequency of singlenouns and compound-nouns that occur independently, namely left and right adjacent words not being nouns. For instance, "word patterns" occurs independently in "... use the word patterns occurring in .... ." Since the scoring functions proposed in 2.2.1 are noun bigram statistics, the number of this kind of independent occurrences of nouns themselves are not used. If we take this information into account, a new type of information is used and better results are expected.

In this paper, we employ a very simple method for this. We observe that if a single-noun or a compound noun occurs independently, the score of the noun is multiplied by the number of its independent occurrences. Then $\mathrm{GM}(\mathrm{CN}, \mathrm{k})$ of the formula (3) is revised. We call this new GM FGM $(\mathrm{CN}, \mathrm{k})$ and define it as follows.

if $\mathrm{N}$ occurs independently

then $\mathbf{F G M}(\mathbf{C N}, \mathbf{k})=\mathbf{G M}(\mathbf{C N}, \mathbf{k}) \times \mathbf{f}(\mathbf{C N})$

where $f(C N)$ means the number of independent occurrences of noun $\mathrm{CN}$

For instance, in example 1, if we find independent "trigram" three times in the corpus, $\operatorname{FGM}($ trigram, 1$)=3 \times \sqrt{(3+1) \times(5+1)}=14.70$ 


\subsubsection{Modified C-value}

We compare our methods with the C-value based method(Frantzi and Ananiadou 1996) because 1) their method is very powerful to extract and properly score compound nouns., and 2) their method is basically based on unithood. On the contrary, our scoring functions proposed in 2.2.1 try to capture termhood. However the original definition of C-value can not score a single-noun because the important part of the definition C-value is:

$\mathrm{C}-\operatorname{value}(\mathrm{a})=($ length $(\mathrm{a})-1)\left(\mathrm{n}(\mathrm{a})-\frac{\mathrm{t}(\mathrm{a})}{\mathrm{c}(\mathrm{a})}\right)$

where $a$ is compound noun, length(a) is the number of single-nouns which make up $a, n(a)$ is the total frequency of occurrence of a on the corpus, $t(a)$ is the frequency of occurrence of $a$ in longer candidate terms, and $c(a)$ is the number of those candidate terms.

As known from (5), all single-noun's $\mathrm{C}$-value come to be 0 . The reason why the first term of right hand side is (length(a)-1) is that C-value originally seemed to capture how much computational effort is to be made in order to recognize the important part of the term. Thus, if the length(a) is 1 , we do not need any effort to recognize its part because the term a is a single-word and does not have its part. But we intend to capture how important the term is for the writer or reader, namely its termhood. In order to make the C-value capture termhood, we modify (5) as follows.

$$
\left.M C \text { - value }(a)=\text { length(a)(n(a) }-\frac{t(a)}{c(a)}\right)
$$

Where "MC-value" means "Modified C-value."

\section{Experimental Evaluation}

\subsection{Experiment}

In our experiment, we use the NTCIR1 TMREC test collection (Kageura et al 1999). As an activity of TMREC, they have provided us with a J apanese test collection of a term recognition task. The goal of this task is to automatically recognize and extract terms from a text corpus which contains 1,870 abstracts gathered from the computer science and communication engineering domain corpora of the NACSIS Academic Conference Database, and 8,834 manually collected correct terms. The TMREC text corpus is morphologically analyzed and POS tagged by hand. From this POS tagged text, we extract uninterrupted noun sequences as term candidates. Actually 16,708 term candidates are extracted and several scoring methods are applied to them. All the extracted term candidates $\mathrm{CNs}$ are ranked according to their $\mathrm{GM}(\mathrm{CN}, \mathrm{k})$, $\mathrm{FGM}(\mathrm{CN}, \mathrm{k})$ and $\mathrm{MC}$-value(CN) in descending order. As for parameter $\mathrm{k}$ of (1) and (2), we choose $k=1$ because its performance is the best among various values of $k$ in the range from 0 to 4 . Thus, henceforth, we omit k from GM and FGM, like $\mathrm{GM}(\mathrm{CN})$ and $\mathrm{FGM}(\mathrm{CN})$. We use $\mathrm{GM}(\mathrm{CN})$ as the baseline.

In evaluation, we conduct experiments where we pick up the highest ranked term candidate down to the PN th highest ranked term candidate by these three scoring methods, and evaluate the set of selected terms with the number of correct terms, we call it CT, within it. In the following figures, we only show CT because recall is CT/8834, where 8834 is the number of all correct terms, precision is CT/PN.

Another measure NTCIR1 provides us with is the terms which include the correct term as its part. We call it "Ionger term" or LT. They are sometimes valued terms and also indicate in what context the correct terms are used. Then we also use the number of longer terms in our evaluation.

\subsection{Results}

In Figure 3 through 5, PN of $X$-axis means PN. 


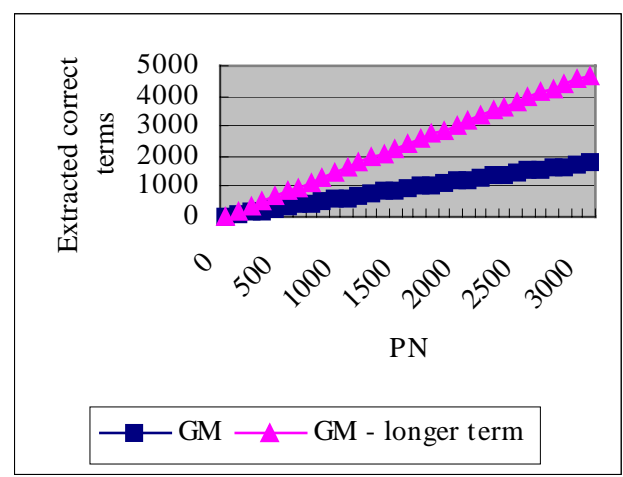

Figure 3. CT and LT of GM(CN) for each PN

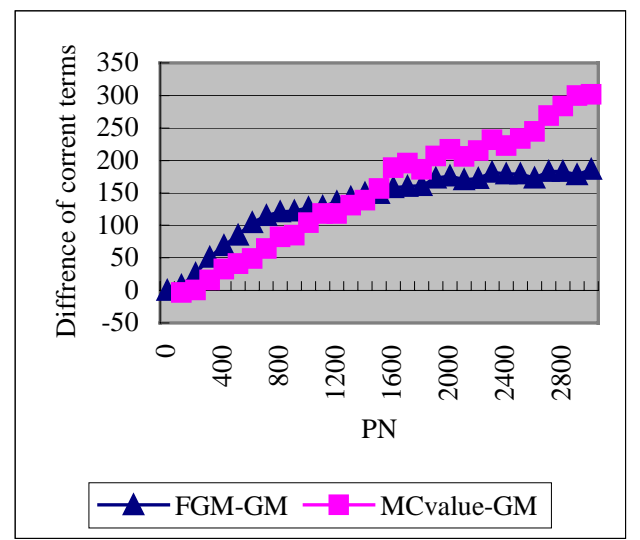

Figure 4. CT of $\operatorname{FGM}(\mathrm{CN})$ minus CT of $\operatorname{GM}(\mathrm{CN})$, and CT of MC-value(CN) minus CT of $\mathbf{G M}(\mathrm{CN})$ for each PN

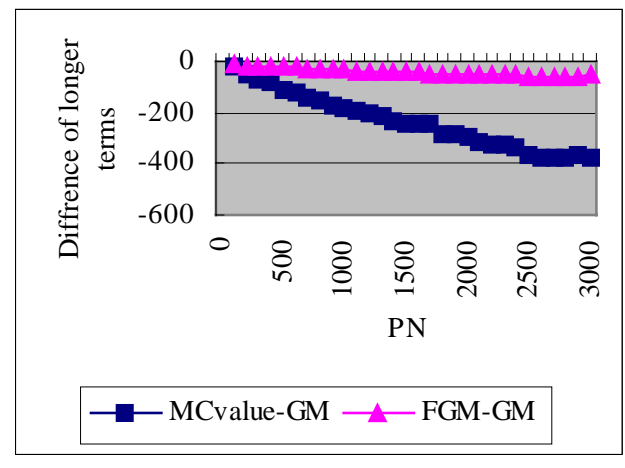

Figure 5. LT of GM(CN) minus LT of FGM(CN), and LT of GM(CN) minus LT of $M C$-value $(\mathrm{CN})$ for each $\mathrm{PN}$

In Figure 3, the $\mathrm{Y}$-axis represents $\mathrm{CT}$ in other words the number of correct terms picked up by GM(CN) and the number of longer terms picked up by $\mathrm{GM}(\mathrm{CN})$ for each PN. They are our baseline. The Figure 4 shows the difference between $\mathrm{CT}$ of $\mathrm{FGM}(\mathrm{CN})$ and $\mathrm{CT}$ of $\mathrm{GM}(\mathrm{CN})$ and the difference between $C T$ of MC-value(CN) and $\mathrm{CT}$ of $\mathrm{GM}(\mathrm{CN})$ for each PN. Figure 5 shows the difference between LT of $\mathrm{GM}(\mathrm{CN})$ and $\mathrm{LT}$ of $\mathrm{FGM}(\mathrm{CN})$ or LT of $M C$-value $(C N)$ for each $P N$. As known from Figure 4, FGM based method outperforms MC-value up to 1,400 highest ranked terms. Since in the domains of TMREC task that are computer science and communication engineering, 1,400 technical terms are important core terms, FGM method we propose is very promising to extract and recognize domain specific terms. We also show CT of each method for larger PN, say, from 3000 up to 15000 in Table 1 and 2.

Table 1. CT of each ranking method for PN larger than 3000

\begin{tabular}{|l|l|l|l|}
\hline PN & GM & FGM & $\begin{array}{l}\text { MC- } \\
\text { value }\end{array}$ \\
\hline 3000 & 1784 & 1970 & 2111 \\
\hline 6000 & 3286 & 3456 & 3671 \\
\hline 9000 & 4744 & 4866 & 4930 \\
\hline 12000 & 6009 & 6090 & 6046 \\
\hline 15000 & 7042 & 7081 & 7068 \\
\hline
\end{tabular}

Table 2. LT of each ranking method for PN larger than 3000

\begin{tabular}{|l|l|l|l|}
\hline PN & GM & FGM & $\begin{array}{l}\text { MC- } \\
\text { Value }\end{array}$ \\
\hline 3000 & 2893 & 2840 & 2531 \\
\hline 6000 & 5644 & 5576 & 5011 \\
\hline 9000 & 8218 & 8152 & 7578 \\
\hline 12000 & 10523 & 10488 & 9852 \\
\hline 15000 & 12174 & 12186 & 12070 \\
\hline
\end{tabular}

As seen in these figures and tables, if we want more terms about these domains, MC-value is more powerful, but when PN is larger than 12,000 , again $F G M$ outperforms. As for recognizing longer terms, $\mathrm{GM}(\mathrm{CN})$, which is the baseline, performs best for every PN. MC-value is the worst. From this observation we come to know that MC-value tends to assign higher score to shorter terms than GM or FGM. We are also interested in what kind 
of term is favored by each method. For this, we show the average length of the highest PN ranked terms of each method in Figure 6 where length of $\mathrm{CN}$ means the number of single-words $\mathrm{CN}$ consists of. Clearly, GM prefers longer terms. So does FGM. On the contrary, MC-value prefers shorter terms. However, as shown in Figure 6, the average length of the MC-value is more fluctuating. That means GM and FGM have more consistent tendency in ranking compound nouns. Finally we compare our results with NTCIR1 results (Kageura et al 1999). Unfortunately since (Kageura et al 1999) only provides the number of the all extracted terms and also the number of the all extracted correct terms, we could not directly compare our results with other NTCIR1 participants. Then, what is important is the fact that we extracted 7,082 correct terms from top 15,000 term candidates with the FGM methods. This fact is indicating that our methods show the highest performance among all other participants of NTCIR1 TMREC task because 1) the highest number of terms within the top 16,000 term candidates is 6,536 among all the participants of NTCIR1 TMREC task, and 2) the highest number or terms in all the participants of NTCIR1 TMREC task is 7,944, but they are extracted from top 23,270 term candidates, which means extremely low precision.

\section{Conclusion}

In this paper, we introduce a new nglenoun bigram based statistical methods for ATR, which capture how many nouns adjoin the singlenoun in question to form compound nouns. Through experimental evaluation using the NTCIR1 TMREC test collection, the FGM method we proposed showed the best performance in selecting up to 1,400 domain specific terms.

\section{Acknowledgements}

This research is funded by the Ministry of Education Science and Academic, J apan.

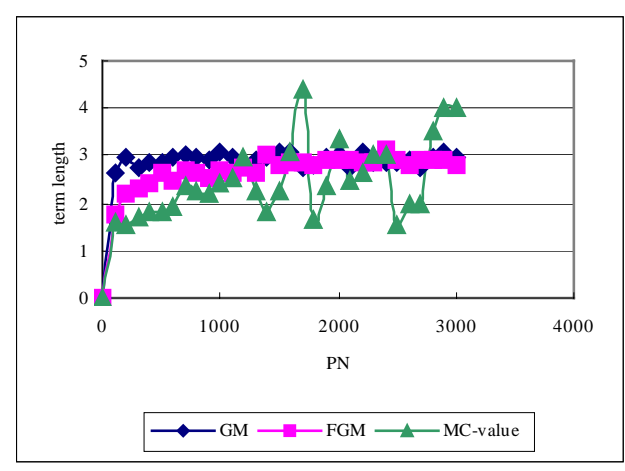

Figure 6. The average length of extracted terms by $\operatorname{GM}(\mathrm{CN})$, $\operatorname{FGM}(\mathrm{CN})$ and $M C$-value $(\mathrm{CN})$ for each $\mathrm{PN}$

References

Frantzi, T.K. and Ananiadou, S. 1996. "Extracting nested collocations". In Proceedings of $16^{\text {th }}$ International Conference on Computational L inguistics, 41-46.

Frantzi, T.K. and Ananiadou, S. 1999. "The c-value/nc-value method for atr". Journal of Natural Language Processing 6(3), 145-179.

Hisamitsu, T, 2000. "A Method of Measuring Term Representativeness". In Proceedings of $18^{\text {th }}$ International Conference on Computational Linguistics, 320-326.

Kageura, K. and Umino, B. 1996. "Methods of automatic term recognition: a review". Terminology 3(2), 259-289.

Kageura, K. et al, 1999. "TMREC Task: Overview and Evaluation". In Proceedings of the First NTCIR Workshop on Research in Japanese Text Retrieval and Term Recognition, 411-440.

Matsumoto, Y., Kurohashi, S., Yamaji, O., Taeki, H. and Nagao, M. 1996. Instruction Manual of Japanese Morphological Analyzer JUMAN3.1. Nagao Lab. at Kyoto University.

Nakagawa, H. and Mori, T. 1998. "Nested Collocation and Compound Noun for Term Recognition". In Proceedings of the First Workshop on Computational Terminology COMPTERM'98, 64-70. 